\title{
Predictive Resource Management for Wearable Computing
}

\author{
Dushyanth Narayanan ${ }^{\dagger}$ and M. Satyanarayanan ${ }^{\dagger \dagger}$ \\ ${ }^{\dagger}$ Carnegie Mellon University and ${ }^{\ddagger}$ Intel Research Pittsburgh \\ \{bumba,satya\}@cs.cmu.edu
}

\begin{abstract}
Achieving crisp interactive response in resource-intensive applications such as augmented reality, language translation, and speech recognition is a major challenge on resource-poor wearable hardware. In this paper we describe a solution based on multi-fidelity computation supported by predictive resource management. We show that such an approach can substantially reduce both the mean and the variance of response time. On a benchmark representative of augmented reality, we demonstrate a $60 \%$ reduction in mean latency and a $30 \%$ reduction in the coefficient of variation. We also show that a history-based approach to demand prediction is the key to this performance improvement.
\end{abstract}

\section{Introduction}

Resource-intensive applications such as speech recognition, language translation, and augmented reality pose a dilemma for wearable computing. Such applications are valuable because they support hands-free interaction. However, their peak resource demands can overwhelm the processing speed, memory, and battery capacity of wearable hardware whose weight, size and form factor are limited by user comfort. The result is sluggish interactive response that can seriously distract a mobile user engaged in a physically and cognitively demanding task such as bridge inspection, aircraft maintenance or military action.

Technology improvements through Moore's Law will not solve this problem. Rather, it is likely to persist because market forces in wearable computing demand continuous improvements in user comfort rather than just improvements in compute power. This tension leads to the question addressed by this paper: How can we achieve crisp interactive response for resource-intensive applications on wearable computers?

In this paper, we show how multi-fidelity computation can help to bound interactive latency by dynamically trading resource demand for output quality, or fidelity. We describe the design, implementation and evaluation of a system that supports multi-fidelity computation. The system automatically makes runtime fidelity decisions on the applications' behalf, thus freeing programmers from this burden. To make sound fidelity decisions, it exploits history-based prediction of application resource usage.

Our implementation is based on Odyssey [27, 12], which originally supported the concept of fidelity for stored data.
This work extends that concept to the broader notion of computational fidelity and demonstrates its applicability to a new class of applications. In the rest of this paper, the term "fidelity" will mean "computational fidelity" and "Odyssey" will refer to the multi-fidelity support added by us to the base system.

We have experimentally validated our approach using four applications. Because of space limitations, we only describe one application case study in detail here, and summarize the results of the other three. Full details of the latter can be found in Narayanan's dissertation [24]. Our key results can be summarized as follows:

- Predictive resource management can bound response latency and reduce its variability.

- History-based prediction of resource demand is feasible, accurate, and necessary for this improvement.

- Legacy applications can be ported at modest cost to a multi-fidelity programming model.

Section 2 describes our high-level design principles and rationale. Section 3 describes our prototype API for multifidelity computation, and the implementation of the runtime support layer. It also explains our methodology for constructing application-specific resource demand predictors, and describes one example in detail. Section 4 presents a comprehensive evaluation of the system: we measure the accuracy of history-based prediction, the performance benefits of predictive resource management, and the programming costs and runtime overheads. Section 5 describes related work, and Section 6 concludes with some directions for future research.

\section{Design rationale}

\subsection{Alternatives}

There are three fundamentally different approaches to coping with situations where application resource demand exceeds supply. One approach is to prevent such situations by using QoS-based resource reservations [23, 19]. For example, an application may be able to reserve a minimum fraction of a CPU and thus guard against insufficient supply of this resource due to competition from concurrent applications. As another example, it may be possible to reserve bandwidth in a carefully controlled networking environment. Unfortunately, enforcement of QoS-based reservations requires op- 


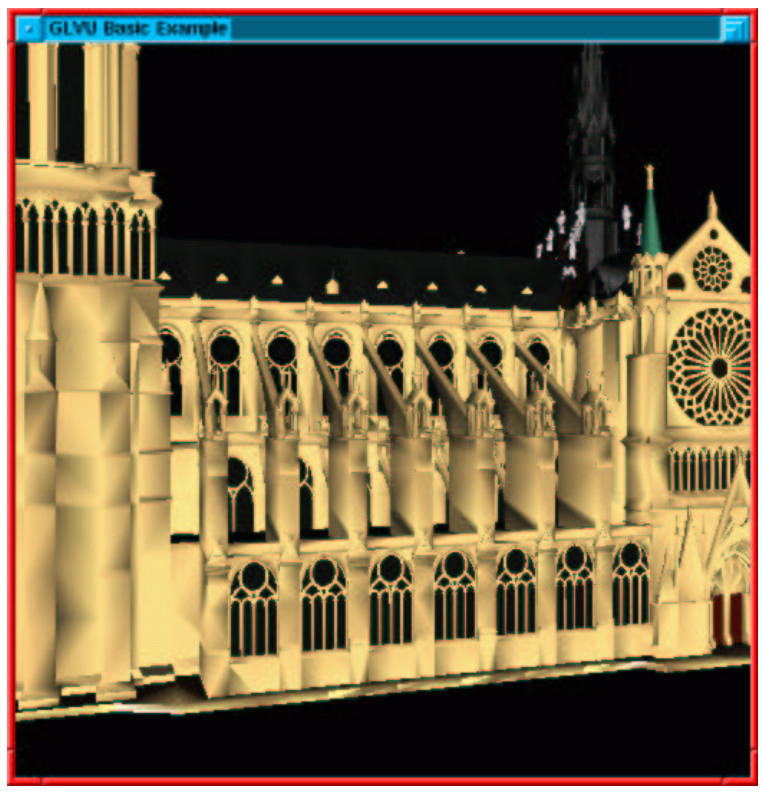

(a) High fidelity (1.0)

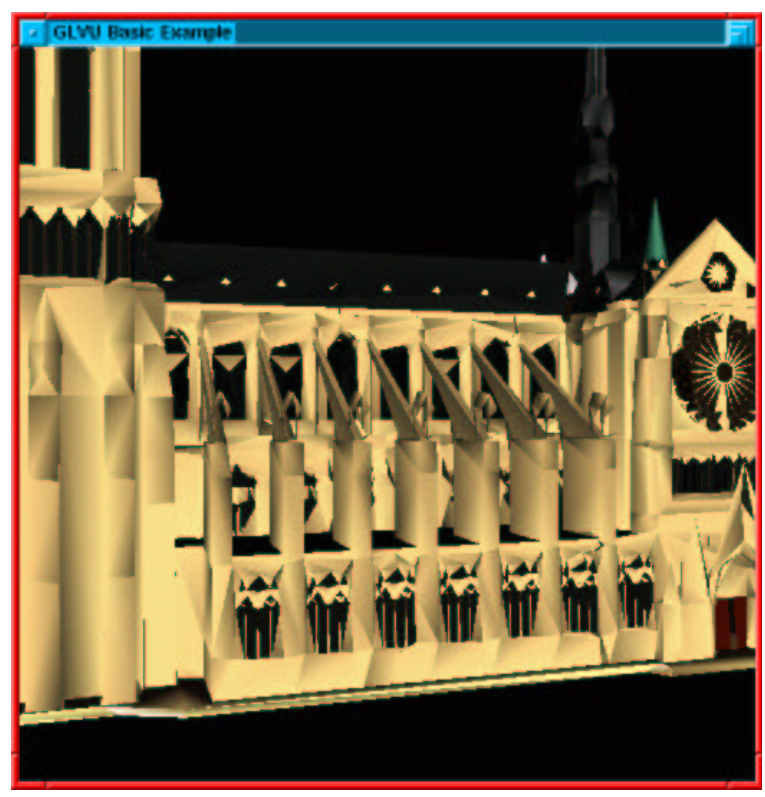

(b) Low fidelity (0.1)

Figure 1: Effect of fidelity on 3-D rendering

erating system support that is rarely present in standard OS distributions. More importantly, this approach fails when the peak resource demand of a single application exceeds the capabilities of the hardware it is running on.

The second approach is to acquire additional resources through remote execution. Even a resource-impoverished wearable computer such as the IBM Linux wristwatch [26] can use compute servers to run resource-intensive applications. We believe that this is a viable strategy, and are indeed exploring it in other research [4]. However, there are many situations in which a mobile user has no access to compute servers and must therefore rely solely on the resources of his wearable computer. A different approach must be used to handle those situations.

The third approach is to reduce resource demand through multi-fidelity computation. As its name implies, multifidelity computation assumes that an application is capable of presenting results at different fidelities. Users prefer results of higher fidelity, but can tolerate results of lower fidelity. A high-fidelity result require greater resources to compute than a low-fidelity result. When resources are plentiful, the application generates high-fidelity results; when resources are scarce, it generates low-fidelity results. By dynamically varying fidelity, timely results can be generated over a wide range of resource levels. We elaborate on this in the next section.

\subsection{Multi-fidelity computation}

The classic notion of an algorithm has a fixed output specification but variable resource demand. In contrast, it is the output specification that is variable in a multi-fidelity computation [30]. By setting runtime parameters called fidelity metrics, we can obtain different outputs for the same input. One can say, in effect, "Give me the best result you can using no more than $X$ units of resource $R . " R$ is typically response latency in an interactive application, but it can also refer to memory, energy, bandwidth or any other resource. Thus multi-fidelity computations are a generalization of anydimension algorithms [22]. The latter can be viewed as multifidelity computations which incrementally refine their output, allowing them to be interrupted at any point to yield a result.

Multi-fidelity computation allows us to choose the best runtime tradeoff between output quality and performance. In an interactive application, each interactive operation can be viewed as a multi-fidelity computation. At the beginning of each operation, its fidelity metrics can be set to yield the desired response latency at the current resource availability.

\subsection{Motivating example}

Throughout this paper we will use augmented reality $(A R)$ [3] as the driving example to illustrate various aspects of our system. Although AR is a relatively young technology, it has already proved useful in a number of domains such as tourist guides [10], power plant maintenance [9], architectural design [34], and computer-supported collaboration [5]. 


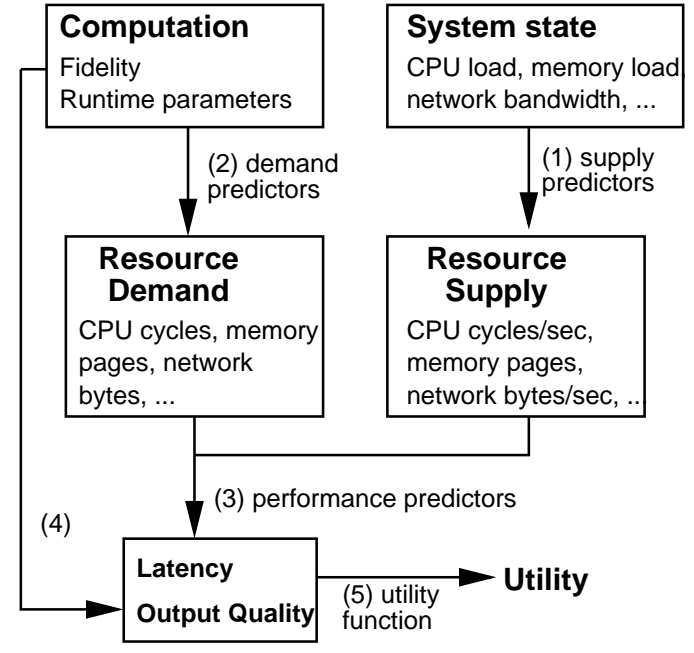

Figure 2: Mapping fidelity to utility

In AR, a user looks through a transparent heads-up display connected to a wearable computer. Any displayed image appears to be superimposed on the real-world scene before the user. AR thus creates the illusion that the real world is visually merged with a virtual world. This requires a precise correspondence between the two worlds. As a user's orientation and location change, the displayed image must rapidly and accurately track those changes. Sluggish tracking can be distracting to the user and, in extreme cases, can result in symptoms similar to sea-sickness.

3-D rendering, a computationally intensive operation, lies at the heart of AR. Even a brief turn of the head by a user can result in a complex scene having to be re-rendered multiple times. For example, an architect might use AR for onsite design. This would allow the architect to visualize the impact of proposed design changes such as new windows or color schemes. Before converging on a final design, he may iteratively try out many alternatives, viewing them from different angles and under different hypothetical lighting conditions such as moonlight or sunset.

3-D rendering for AR embodies many of the characteristics that motivate the work described in this paper. First, it is extremely resource intensive, particularly of CPU and memory. Second, to be fully effective it must run on a lightweight wearable computer. Third, crisp interactive response is critical. Fourth, there is a fidelity metric, the resolution of the displayed image, that directly impacts resource consumption.

Figure 1 illustrates the last point. The high-fidelity figure on the left contains ten times as many polygons as the lowfidelity figure on the right. Since CPU demand increases with the number of polygons, the low-fidelity figure can be rendered much faster. In many situations, the low-fidelity figure may be acceptable; the user can always explicitly ask for rerendering at higher fidelity.

\subsection{Predictive resource management}

Before executing an interactive operation, an application must determine its fidelity settings. Odyssey serves as an oracle in making this decision. Its recommendation is based on a search of the space of fidelity settings. This search requires Odyssey to predict resource supply during the operation, as well as resource demand and operation latency for different settings. It also requires Odyssey to correctly reflect the user's current preferences in the tradeoff between output quality and operation latency.

As Figure 2 shows, the complete prediction process can be decomposed into five predictive mappings. Three of the mappings relate to predicting operation latency: (1) from system load statistics to resource supply; (2) from fidelity to resource demand; and, (3) from resource supply and demand to operation latency. The other two mappings translate fidelity and latency predictions into predictions of user satisfaction or utility: (4) from fidelity to output quality; and, (5) from latency and output quality to utility.

Odyssey performs mapping 1 using supply predictors that monitor kernel load statistics through standard interfaces, and make inferences based on gray-box knowledge [2] of kernel resource management policies. It uses history-based demand predictors to perform mapping 2, and performance predictors based on a resource model for mapping 3. These components are described in Sections 3.3 and 3.4.

Mapping 4 specifies the output quality we can expect at each setting of each fidelity "knob". In general, this mapping would be determined through studies of user perception. In this work, we make the simplifying assumption that fidelity and output quality are synonymous; in other words, that the mapping is trivial. Mapping (5) is a utility function that captures current user preferences. In a deployed system, utility functions would be generated automatically from a GUI or by inferring user intent. In our experimental prototype, we use the hand-crafted utility functions described in Section 3.5.

\section{Interface and implementation}

\subsection{Programming interface}

The multi-fidelity programming model is based on the notion of an operation. An operation is the smallest user-visible unit of execution, from user request to system response. Each operation corresponds to one multi-fidelity computation, with fidelity metrics settable at operation start. Examples include rendering an augmented reality scene; recognizing a speech utterance and displaying the recognized text; fetching and displaying a web image.

Figure 3 shows the basic multi-fidelity API. register_fidelity is called at application startup. Odyssey then reads an Application Configuration File (ACF), which specifies the multi-fidelity operation type, its fidelity metrics, and their value ranges (Figure 4). 


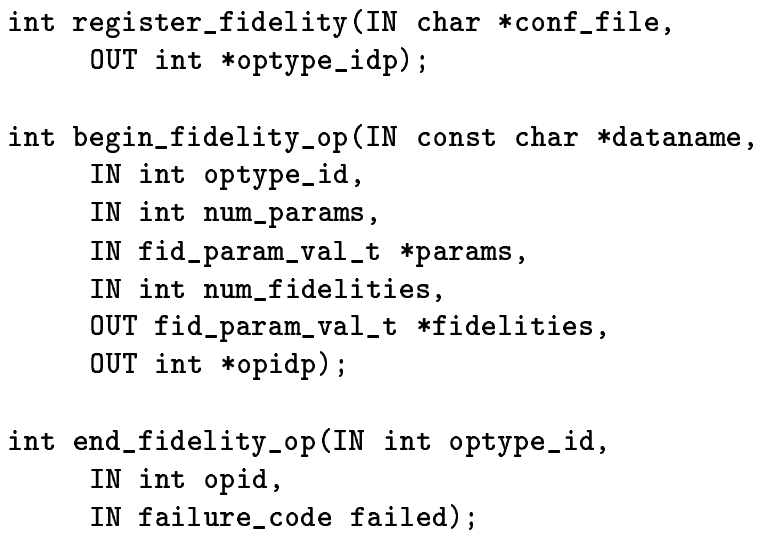

C function prototypes for the API described in Section 3.1.

Figure 3: The Odyssey multi-fidelity API

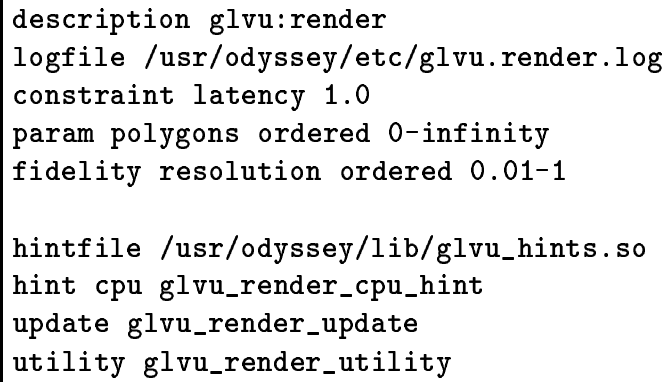

Example of ACF described in Section 3.1.

Figure 4: Application Configuration File for rendering

The ACF also specifies nontunable parameters: runtime variables such as input data size that affect resource demand, but are not adaptable. For example, the resource demand of rendering depends not only on the resolution, but also on the polygon count of the original, full-resolution scene.

Finally, the ACF specifies an application-specific hint module. This binary module contains the application-specific resource demand predictors and the user utility function. For efficient runtime invocation of the demand predictors and utility function, the hint module is loaded into Odyssey's address space. We are looking at ways to retain the efficiency, but improve on the safety, of this approach.

Before each operation, the application invokes begin_fidelity_op, and passes in the nontunable parameters. Odyssey computes and returns the optimal fidelity value(s) for the operation. After each operation, the application calls end_fidelity_op. Odyssey then logs the operation's measured resource demand: these logs are used for history-based resource demand prediction (Section 3.4).

\subsection{System architecture}

Odyssey is implemented as a user-level process on a standard Linux 2.4 kernel. Its primary functionality - making fidelity decisions - is triggered by begin_fidelity_op and implemented in the following steps, numbered as in Figure 5:

1. The application passes in the nontunable parameters.

2. Supply predictors estimate the application's resource supply for the near future (mapping 1 of Figure 2).

3. An iterative solver searches the fidelity space for the best candidate.

4. Demand predictors map fidelity to resource demand (mapping 2).

5. A performance predictor estimates latency given supply and demand predictions (mapping 3).

6. A utility function evaluates the proposed fidelityperformance tradeoff (mapping 5).

7. After several iterations of steps $3-6$, the solver returns the fidelity with the highest utility.

The system's second function - monitoring and logging — is triggered by end_fidelity_op:

8. Demand monitors measure the resources consumed by the just-concluded operation.

9. A logger records the resource demand, fidelity, and nontunable parameter values to a disk file.

10. These values are also passed to the demand predictors, to update their predictive models.

Section 3.3 describes the generic system components: the supply predictors, performance predictors, solver, demand monitors, and logger. Demand predictors are applicationspecific: Section 3.4 describes our history-based method for constructing them. Section 3.5 then describes our approach to constructing utility functions.

\subsection{Generic system components}

\subsubsection{Supply predictors}

Our prototype has supply predictors for CPU, memory, network, energy and file cache. Each of these monitors kernel statistics, and makes predictions of resource availability for each application at the beginning of each operation. For brevity, we only describe the CPU supply predictor here.

The CPU supply predictor predicts, at the start of each operation, the CPU supply available to it in cycles/sec. It is based on some simplifying assumptions: that the operation is single-threaded, that all CPU-bound processes receive equal shares (and that I/O-bound processes offer negligible CPU load), and that past load predicts future load at all time scales. These assumptions give us a simple predictor: a process $p$ 's CPU supply over the next $T$ seconds is

$$
S_{\text {cpu }}=\frac{P}{N+1}
$$

where $P$ is the processor clock speed, and $N$ is the predicted background load over the next $T$ seconds: that is, the average 


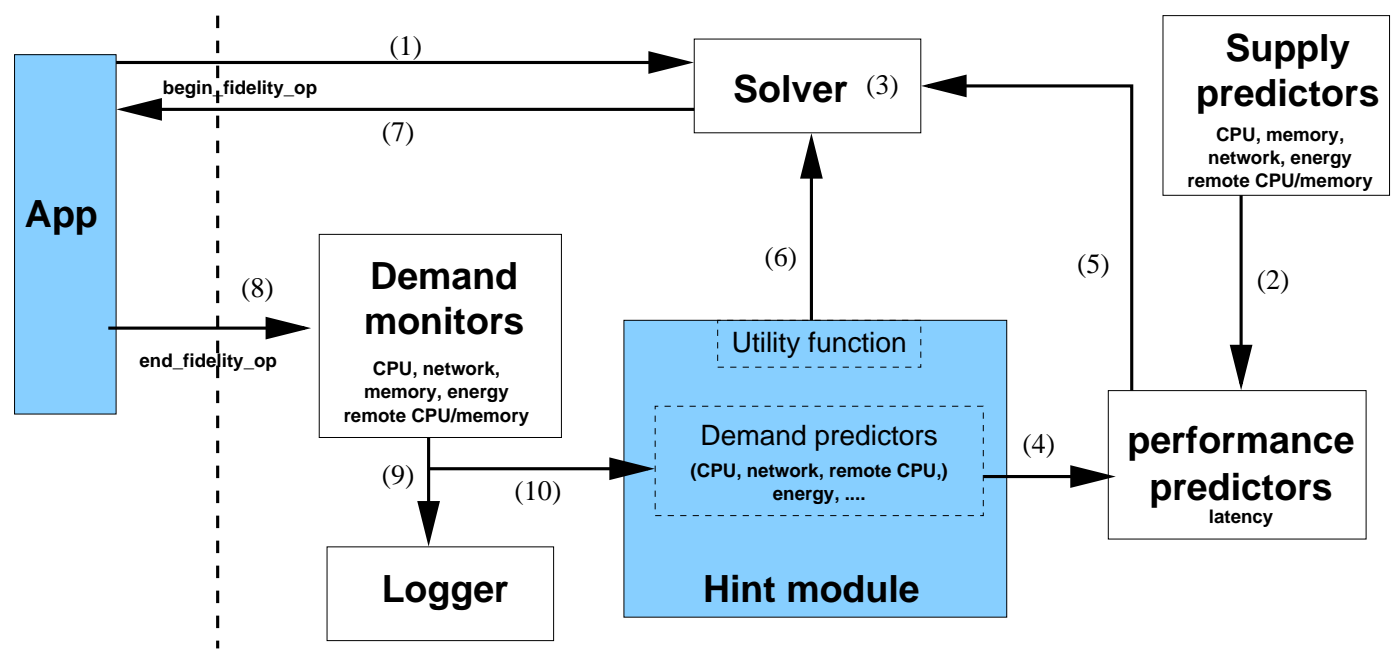

Shaded boxes represent application-specific components; components to the right of the dotted line are part of Odyssey. The numbers correspond to the steps in Section 3.2.

Figure 5: System support for the multi-fidelity API

number of runnable processes other than $p$. We periodically sample the instantaneous load average $n_{i}$ from/proc/loadavg, and subtract out $p$ 's contribution, $n_{i}(p)$. The latter is 1 if $p$ is runnable, and 0 if not. We then smooth the samples:

$$
N_{i+1}=\alpha N_{i}+(1-\alpha)\left(n_{i}-n_{i}(p)\right)
$$

Here $t_{p}$ is the load sampling period, $0.5 \mathrm{~s}$ in our prototype. The constant $\alpha=e^{\frac{-t_{p}}{T}}$ makes the decay time equal to the prediction horizon $T$. In other words, we use more history for predictions over longer periods.

The clock speed $P$ is read from /proc/cpuinfo at startup. Currently Odyssey runs on a stock Linux kernel without dynamic clock scaling support. When such support is available, it should be possible to update $P$ dynamically from /proc whenever the clock speed changes.

\subsubsection{Performance predictors}

Our current prototype has predictors for two performance metrics: operation latency and battery drain [12]. Here we focus on operation latency, the key metric for interactive applications.

Our latency predictor computes latency as a function of resource supply and demand. It is based on a simple resource model that assumes sequential use of resources (no overlapping of processing and network $\mathrm{I} / \mathrm{O}$ ). it computes latency as:

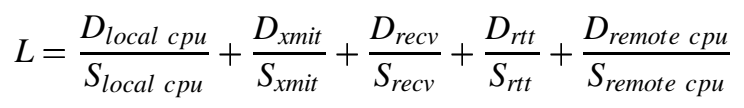

Here $S_{\text {local cpu }}$ is the predicted CPU supply in cycles/sec available to the application. $D_{\text {local cpu }}$ is the predicted CPU demand in cycles required by the operation. The other terms represent the latency of transmitting data to a remote compute server, receiving data, round-trip time, and computation done at the server.

The predictor also factors in the latency cost of using other resources, such as virtual memory paging or remote server access [24]. For brevity, we do not discuss these.

The default generic latency predictor can be overridden at runtime by an application-specific predictor: for example, one that allows for overlapping computation and I/O.

\subsubsection{Solver, demand monitors, and logger}

The solver searches the space of fidelities and finds the values that maximize utility. It uses a gradient-descent strategy for numeric parameters, and exhaustive search for non-numeric parameters such as enumerated lists. It works well for applications with a small number of fidelity metrics and wellbehaved utility functions without multiple local maxima; we could easily substitute more robust and scalable algorithms such as Lee's [19] without modifying other system components.

Demand monitors measure the resource demand of each operation based on kernel statistics from /proc. For example, CPU demand is the CPU time used by an operation, scaled by the processor clock speed. This information is written to a disk file by the logger.

\subsection{History-based demand predictors}

A key component of our architecture is the demand predictor: a function that maps an operation's fidelities and nontunable parameters to its resource demand, in units independent of runtime system state such as load or clock speed (for example, CPU demand is measured in cycles consumed per operation). 


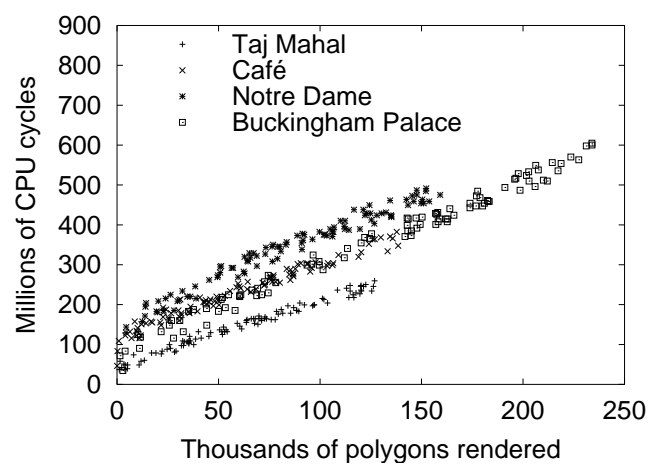

The graph shows the CPU demand of rendering for four different scenes at different resolutions (fidelities). For each scene, the camera position was fixed arbitrarily. All experiments were run on the hardware described in Section 4.1.

Figure 6: CPU demand of rendering as a function of fidelity and scene

We construct demand predictors empirically from application history logs [25], rather than relying exclusively on static analysis. First, the application programmer or domain expert identifies fidelity metrics and other runtime parameters affecting resource demand. From a static analysis, they might also give a functional form relating these parameters to resource demand: for example, "CPU demand is quadratic in input data size".

The remaining steps are automated, requiring little or no user intervention: we run the computation at different parameter values, and Odyssey automatically logs each operation's resource demand. We use statistical machine learning techniques to fit the logged data to the functional form, generating a predictive mapping function. At runtime, we continue to refine this function using online learning techniques.

Although demand predictors are application-specific, we believe our methodology will allow their construction by third parties without extensive domain expertise. Additionally, demand predictors are separate code modules, and do not require modification of the application source code. We illustrate our method through one detailed example, and describe two techniques that proved extremely useful in improving predictor accuracy. Section 4.3 evaluates prediction accuracy for our chosen example as well as for other applications and resources.

\subsubsection{Example: CPU demand predictor for rendering}

Rendering is CPU-bound, and good interactive response depends on accurate prediction and regulation of CPU demand. For our rendering algorithm, resolution is the fidelity metric: thus we need to know the mapping from resolution to CPU demand. CPU demand depends both on the resolution $r$ and the original polygon count $p$; from examining the algorithm, we expected in fact that it would be a function of the rendered polygon count $p r$.
To map resolution to CPU demand, we started by logging the CPU demand at different resolutions for four different scenes, and plotting CPU demand against rendered polygon count (Figure 6). We see that CPU demand is linear in rendered polygon count:

$$
D_{c p u}=c_{0}+c_{1} p r
$$

for a fixed scene and camera position (note that different scenes have different values of $c_{0}$ and $c_{1}$ ) and a fixed camera position. However, the scene and the camera position are parameters that can vary at runtime, and must be tracked. In the following sections, we show how we track this variation using data-specific prediction and online learning.

\subsubsection{Data-specific prediction}

Sometimes resource demand depends on data-specific effects other than the data size, which are not easily expressed as numeric parameters. For example, the CPU demand of rendering depends on the contents of the scene being rendered. In such cases, data-specific prediction can be extremely useful: maintaining separate predictor coefficients for each data objects. Sometimes, these can be computed offline and stored with the data: for example, JPEG [33] compression ratios depend on image content, and these "compressibility coefficients" could be precomputed and stored at the web server.

In other cases, the data-specific coefficients must be computed online, after observing the resource demand of a few operations on a new data object. This can still be useful if we perform many operations on the same object: for example, with rendering, the user will usually navigate a single scene for a while. We start with a generic linear predictor computed over a number of test scenes. When a new scene is loaded, we initialize a new predictor, initially identical to the generic predictor. After each render, we update the predictor with the measured resource demand to specialize it for the scene.

\subsubsection{Online learning}

Sometimes, we may have portions of application state which affect resource demand but are not easily used as part of a predictive model. For example, the CPU demand of rendering depends not only on the resolution and the scene, but also on the camera position. Figure 7 shows that the CPU demand of rendering varies considerably with camera position as a user navigates a scene, even when fidelity is fixed.

Thus, camera position and orientation are nontunable parameters affecting CPU demand. Unfortunately, their effect on CPU demand is very complex, depending on local properties of the scene: mapping them directly to CPU demand requires large and expensive lookup tables. Instead, we use a much simpler technique based on the observation that

- At each camera position, the linear relationship $D_{c p u}=$ $c_{0}+c_{1} p r$ holds, but $c_{0}$ and $c_{1}$ vary with camera position.

- In typical use, camera position changes incrementally: the user follows a continuous path through the scene. 


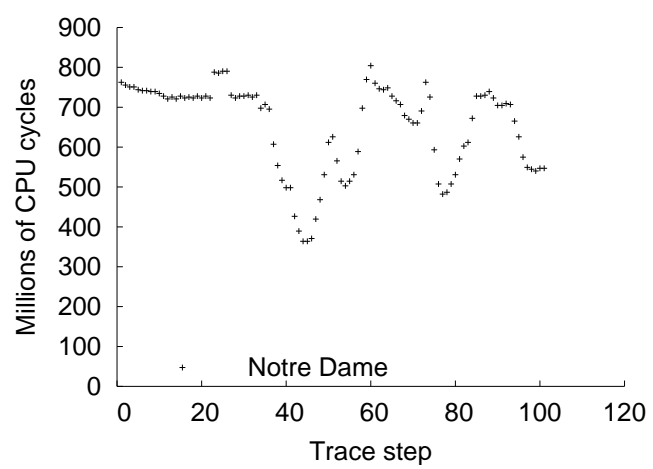

The graph shows the CPU demand of rendering the Notre Dame scene over time, at a fixed resolution of 1 . Each point corresponds to one camera position in a motion trace of a user navigating the scene.

Figure 7: CPU demand of rendering: fixed resolution, moving camera

- CPU demand has locality: a small change in camera position results in a small change to $c_{0}$ and $c_{1}$.

We use an online-learning method that uses the linear mapping $D_{c p u}=c_{0}+c_{1} p r$, but continuously updates the values of $c_{0}$ and $c_{1}$ to reflect the behaviour corresponding to the current camera position. We use recursive least-squares regression with exponential decay [36], a modification of the well-known linear regression method [15]. This gives greater weight to more recent data by decaying the weight of data exponentially over time. Our predictor uses a decay factor of 0.5 , which makes it very agile, effectively remembering only the last 4 data points. It is also cheap, requiring (for a 2-dimensional linear fit) only tens of bytes of state, and tens of floating point instructions per update.

The online-learning predictor also tracks data-specificity. For each new scene, we create a new predictor that is initialized with the generic coefficients: subsequent renders of that scene result in updates of the scene-specific predictor, specializing it both for the scene and the camera position within the scene. Section 4.3 shows that these two simple techniques improve prediction accuracy significantly for rendering; we believe that they have more general applicability as well.

\subsection{Utility functions}

Utility functions represent a user's tradeoff policy between fidelity and performance. Given some estimated fidelity and performance, the utility function returns a number in $[0,1]$ representing the resulting user happiness; 0 represents the least possible user satisfaction and 1 the most. By default, we use linear functions for utility as a function of fidelity, and sigmoids for utility as a function of latency. The product of these functions gives us a multidimensional utility function whose range is still $[0,1]$. In Odyssey, utility functions are computed by binary code modules; the user can override the default utility function with an arbitrarily general one by pro-

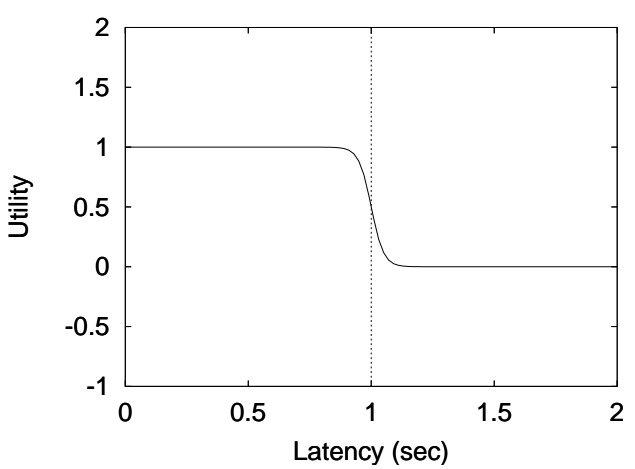

Figure 8: Sigmoid utility function

viding their own module.

A sigmoid is a smoothed version of a step function. Instead of having utility fall off a cliff when latency exceeds its target value, we can now specify a tolerance zone where latency degrades linearly. Figure 3.5 shows a sigmoid with a target of $1 \mathrm{~s}$ and a tolerance of $10 \%$. There is little gain in utility from decreasing latency below $0.9 \mathrm{~s}$ : this is the sweet spot of the curve. Above $0.9 \mathrm{~s}$, utility decreases steadily, and latencies above $1.1 \mathrm{~s}$ are unacceptable to the user. $\mathrm{M}$

\section{Evaluation}

This section validates the predictive resource management approach by answering three sets of questions:

- Is history-based demand prediction accurate? Are dataspecific prediction and online learning useful?

- What are the performance benefits of predictive resource management? Can multiple, concurrent adaptive applications improve their performance without interfering with each other?

- What are the programming costs and runtime overhead of using the system?

Before we describe the experiments that answer these questions, we first describe our experimental setup (Section 4.1) and evaluation metrics (Section 4.2). Sections 4.3-4.5 then address each of the above questions in turn.

\subsection{Experimental platform and benchmarks}

Our platform for all experiments reported in this paper is an IBM ThinkPad 560 with a $233 \mathrm{MHz}$ Mobile Pentium MMX processor, $96 \mathrm{MB}$ of RAM, no 3-D graphics hardware, and running a standard Linux 2.4.2 kernel. We used this rather than a wearable computer for ease of development and testing; its processing power is comparable with recent wearable and handheld platforms such as the IBM Linux watch [26] and the Compaq iPAQ 3650.

Our motivating example - augmented reality — is not a mature technology, and fully fledged AR applications are not 
freely available. Instead, we use as benchmarks two applications - GLVU and Radiator - which provide one component of AR: 3-D rendering. Together, these applications approximate the augmented reality scenario of Section 2.3: an architect using AR for on-site design.

GLVU [29] is a "virtual walkthrough" program that allows a user to explore a virtual 3-D scene: its function is to render the scene from any viewpoint chosen by the user. In our experiments, we simulate a moving user by replaying a trace of a user navigating a 3-D scene using GLVU's graphical user interface. We assume a continually moving user, and do not insert any think times between render requests.

Radiator [35] computes lighting effects for 3-D rendering using radiosity algorithms [6]. In an AR scenario, it would be re-run whenever the user modified the scene lighting, for example by adding a window to a building. We simulate this user behaviour by running sporadic radiosity computations during the virtual walkthrough, with random intervening think times.

Both GLVU and Radiator support multiresolution scaling [14], which allows each render or radiosity computation to be done at any resolution - any fraction of the original polygon count. The overhead of changing the resolution is negligible. Resolution is thus the single fidelity metric for both computations.

In a real AR application, the user would be able to interactively edit the scene, and the lighting effects computed by Radiator would be fed back into GLVU for rendering. In our version, GLVU and Radiator lack interactive editing facilities and do not communicate with each other. However, the benchmarks are representative of AR from a resource and performance point of view.

\subsection{Evaluation metrics}

Demand predictor accuracy is measured by running an application benchmark on an unloaded system, and comparing the predicted resource demand for each operation with the actual, logged value. We use relative rather than absolute prediction error, since it is applicable across a wide range of values. Given the relative error for a number of operations, we report the 90th percentile error $E_{90}$. An $E_{90}$ of $5 \%$ means that $90 \%$ of the time, the predictor was within $5 \%$ of the correct value.

Our metric of interactive application performance is operation latency. Specifically, we measure Odyssey's ability to keep latency within user-specified bounds, with low variability and without unnecessarily sacrificing fidelity. In other words, we measure the ability of the adaptive mechanism Odyssey — to implement one kind of policy: keeping latency steady. Our adaptive policies are implemented by a sigmoidal utility function centred on the desired latency bound (Section 3.5), with a tolerance of $10 \%$. Utility also increases linearly with fidelity. The net effect is that utility is maximized at $90 \%$ of the latency bound: this is the target latency.

We conduct 5 trials of each experimental run. For each such set of 5 trials, we report the mean operation latency, and

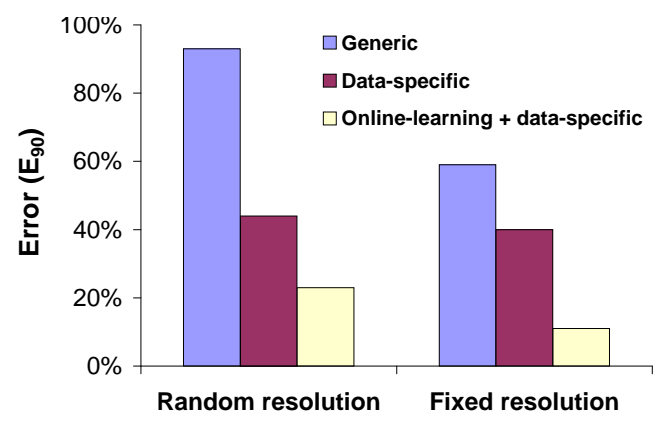

We show 90th percentile error (in \%) of three different schemes for predicting the CPU demand of rendering. The CPU demand itself varies between $0.1 \mathrm{~s}$ and $5 \mathrm{~s}$ (23-1018 million cycles).

Figure 9: CPU demand prediction error for rendering

also the coefficient of variation: the standard deviation of latency divided by the mean. In some cases, we also show a timeline of one of the trials, to illustrate the performance and fidelity over time.

Ideally, we want mean latency to be on target. Higher latencies indicate bad interactive response, while lower latencies indicate an unnecessary sacrifice of fidelity. We also want the coefficient of variation to be small: variability in performance leads to a bad user experience [21]. High variation also indicates that the system is often off-target: in other words, not implementing the adaptive policy well.

\subsection{Demand predictor accuracy}

In this section, we show that history-based demand predictors provide accurate predictions across a range of applications and resources. For brevity, we describe in detail only the CPU demand predictor for GLVU, and summarize results for other predictors.

For GLVU, we measured the accuracy of the data-specific, online-learning predictor, and also the contribution of dataspecificity and online learning to this accuracy. We compared

- a generic predictor, which fits a single pair of coefficients $c_{0}, c_{1}$ to all 4 scenes,

- a data-specific predictor, which specializes $c_{0}$ and $c_{1}$ to each scene,

- the online-learning predictor, which maintains scenespecific coefficients, and also updates them after each operation to track runtime variation in CPU demand.

CPU demand depends not only on camera position, but also on fidelity. The accuracy of prediction depends on the amount of variation in fidelity, which depends on the variation in resource supply at runtime. To estimate demand prediction accuracy independent of runtime conditions, we evaluated both the worst case — randomly varying fidelity — and the best case - fixed fidelity.

Figure 9 shows the prediction error of these three predictors for both random and fixed resolution (1.0), measured on 


\begin{tabular}{|c|c|c|c|c|c|c|c|}
\hline Application & $\begin{array}{l}\text { Tunable } \\
\text { parameters }\end{array}$ & Resource & $\begin{array}{l}\text { Observed } \\
\text { of resource }\end{array}$ & $\begin{array}{l}\text { range } \\
\text { demand }\end{array}$ & $\begin{array}{l}\text { Data } \\
\text { specific? }\end{array}$ & $\begin{array}{l}\text { Online } \\
\text { learning? }\end{array}$ & $E_{90}$ \\
\hline GLVU & Resolution & CPU & $23-1018$ & Mcycles & Yes & Yes & $24 \%$ \\
\hline \multirow[t]{2}{*}{ Radiator } & \multirow{2}{*}{$\begin{array}{l}\text { Resolution, } \\
\text { algorithm }\end{array}$} & Memory & $14-60$ & MB & No & No & $3 \%$ \\
\hline & & CPU & $220-46219$ & Mcycles & Yes & No & $11 \%$ \\
\hline Web browser & JPEG level & Energy & $1.5-25$ & Joules & Yes & No & $9 \%$ \\
\hline \multirow{3}{*}{$\begin{array}{l}\text { Speech } \\
\text { recognizer }\end{array}$} & \multirow{3}{*}{$\begin{array}{l}\text { Client-server } \\
\text { split, vocab. } \\
\text { size }\end{array}$} & Network & $4-219$ & KB & No & No & $0.3 \%$ \\
\hline & & client CPU & $0-2774$ & Mcycles & No & No & $10 \%$ \\
\hline & & server CPU & $0-2128$ & Mcycles & No & No & $16 \%$ \\
\hline
\end{tabular}

The table shows the 90th percentile error $E_{90}$ (right-most column) of history-based demand predictors for different applications and resources. In each case, we also show the observed min-max range of resource demand, measured in millions of cycles of CPU, megabytes of memory, Joules of energy, or kilobytes of network transmission/reception.

Figure 10: Demand predictor accuracy for various applications and resources

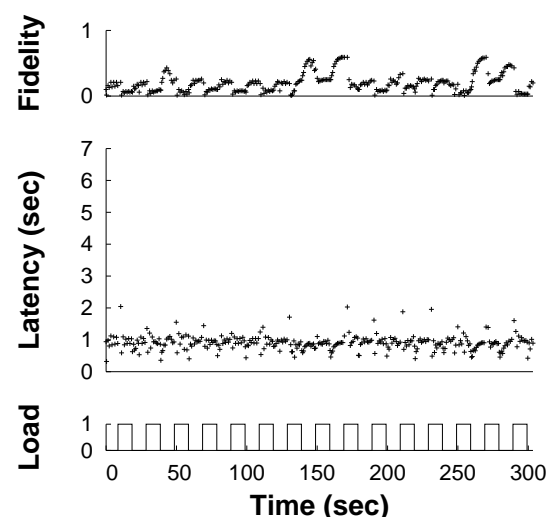

(a) Fully adaptive
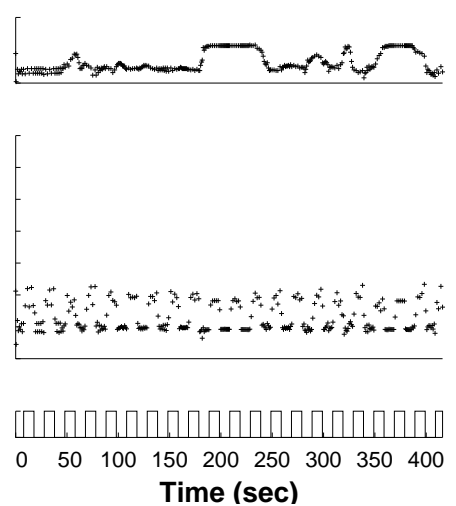

(b) Demand-only
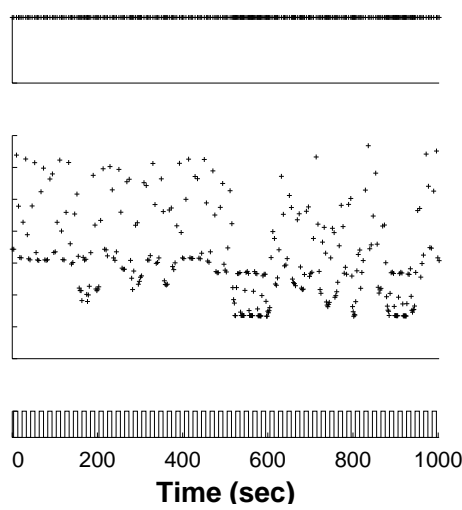

(c) Static

Fidelity and latency of GLVU over time when subjected to a time-varying background load, in three different adaptation configurations. Note the different time scales on the $x$ axes: the same benchmark takes different amounts of time in different configurations.

Figure 11: Adaptation in GLVU

user traces on four different scenes. Each trace has 100 camera positions, yielding 400 data points in all. We see that both data-specificity and online learning decrease prediction error: the best predictor - online-learning - has an error of $24 \%$ in the worst case. This is small compared to the orderof-magnitude variation in CPU demand; better learning techniques could probably reduce error further.

We also measured demand predictor accuracy for other applications - Radiator, speech recognition, and web browsing - and other resources - memory, network, and battery energy (Figure 10). In each case, we are able to predict to within a small fraction a quantity with a large dynamic range, showing that multi-fidelity computation can make a big difference to resource demand, and that we can predict resource demand to within a small error. Note that all the other predictors have better accuracy than the CPU predictor for GLVU: our chosen example case study represents our worst observed case.

\subsection{Performance benefits}

\subsubsection{Single application with background load}

Given that demand prediction is accurate, what is the impact on performance of predictive resource management? To answer this question, we measured the performance of GLVU adapting dynamically to changes in resource supply. GLVU plays a trace of a user navigating the "Notre Dame" scene, while Odyssey attempts to bound operation latency to $1 \mathrm{~s}$. Simultaneously, a competing process alternates every $10 \mathrm{~s}$ between spinning the CPU and sleeping. We chose this square waveform over more realistic load patterns to explore the effect of different load frequencies and amplitudes.

We ran this experiment in three configurations:

- Fully adaptive: both supply and demand prediction are enabled, so GLVU adapts to changes both in application demand and in background load. 


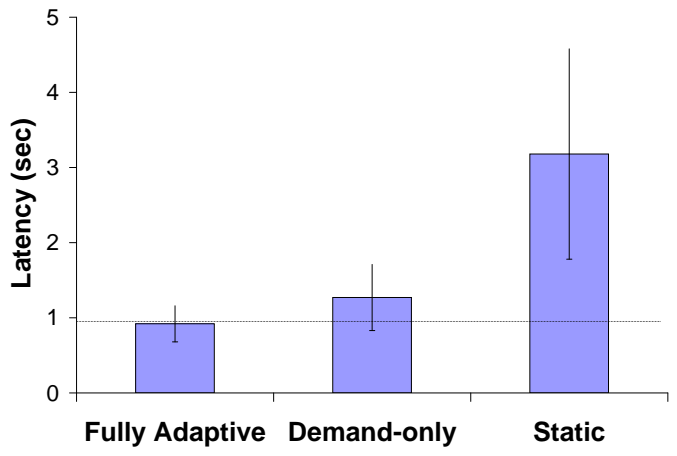

(a) Mean latency

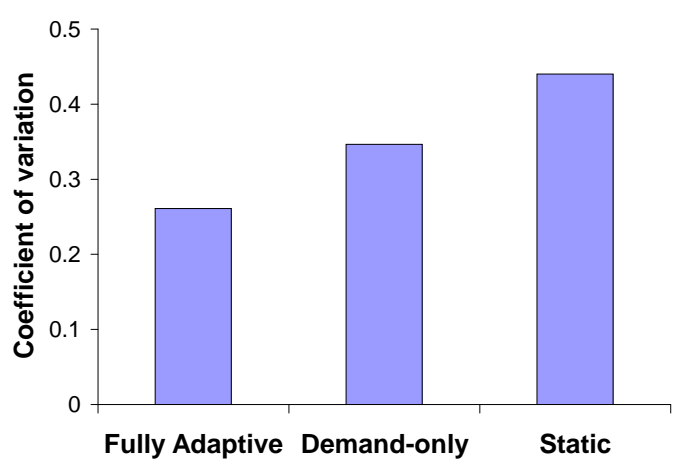

(b) Variability in latency

Error bars show standard deviations; the horizontal line marks the target latency.

Figure 12: Adaptive performance in GLVU

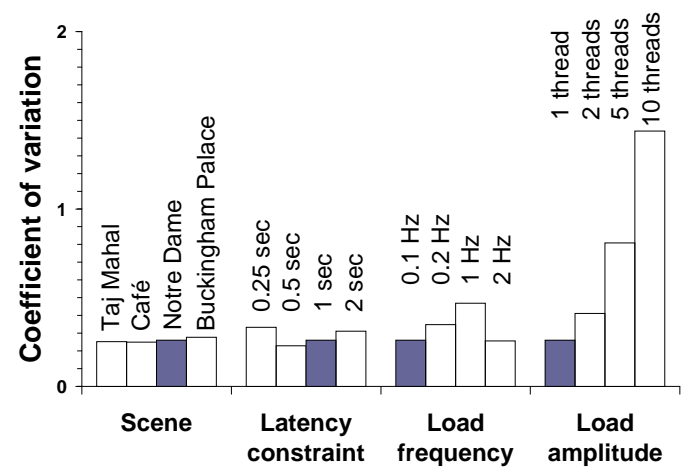

The graph shows the coefficient of variation for latency under various experimental conditions. Each set of bars varies one parameter: the shaded bars represent the baseline case.

Figure 13: GLVU adaptation: sensitivity analysis
- Demand-only: we enable CPU demand prediction, which allows GLVU to regulate its CPU demand to the target value. However, supply prediction is disabled: the background load is assumed to be 0 .

- Static: GLVU's fidelity is fixed at 1: there is no adaptation at all.

Figure 11 shows one run for each configuration, in our baseline case: a trace of "Notre Dame" with a $1 \mathrm{~s}$ latency bound, a $0.1 \mathrm{~Hz}$ background load frequency, and a peak load of 1 . We see that with the "fully adaptive" keeps latency on target. "Demand-only" is on target only when unloaded, and "static" almost never. Note that the different experiments have different run times, the effect of reducing mean latency on a work-based benchmark.

Figure 12 shows mean latency and variation over 5 trials for each configuration. We see that demand prediction alone substantially improves interactive performance by bringing mean latency close to the target value and reducing variability; enabling supply prediction improves performance further.

To validate our results across a range of experimental parameters, we tested the "fully adaptive" configuration with different 3-D scenes, latency bounds, and load patterns. In each case, we varied one parameter, keeping the others fixed, and compared the performance against the baseline case: Figure 13 shows the results of these experiments.

Mean latency was insensitive to experimental parameters, except when we reduced the latency bound to $0.25 \mathrm{~s}$ : in this case mean latency exceeds target by $20 \%$. Here we hit the limit of fidelity degradation: on our test platform, rendering can take up to $0.46 \mathrm{~s}$ of CPU time even at the lowest fidelity.

Variability in latency was the same for all scenes, but varied with other parameters. Variability was lowest for a $0.5 \mathrm{~s}$ latency bound. At lower latencies, scheduler effects (Linux's $200 \mathrm{~ms}$ quanta) cause variability. At higher latencies, load transitions are more frequent with respect to operation rate, causing more operations to deviate from target.

Variability was highest when load frequency matched operation rate $(1 \mathrm{~Hz})$. At lower frequencies, fewer operations are hit by load transitions. At higher frequencies, load variation gets smoothed out over the course of an operation. Variability also increases sharply with increasing load amplitude (peakto-trough difference): operations hit by load transitions are more affected by larger transitions.

We observe that it is most important to predict resource supply at the time scale of adaptation: higher and lower frequencies impact latency less. If this time scale is comparable to the scheduler granularity, then prediction accuracy will be low and performance variability will be high.

\subsubsection{Concurrent applications}

When we run two adaptive applications concurrently, are they both able to adapt effectively, or do they interfere with each other's performance? To answer this question, we mimicked an AR scenario by running GLVU and Radiator concurrently 

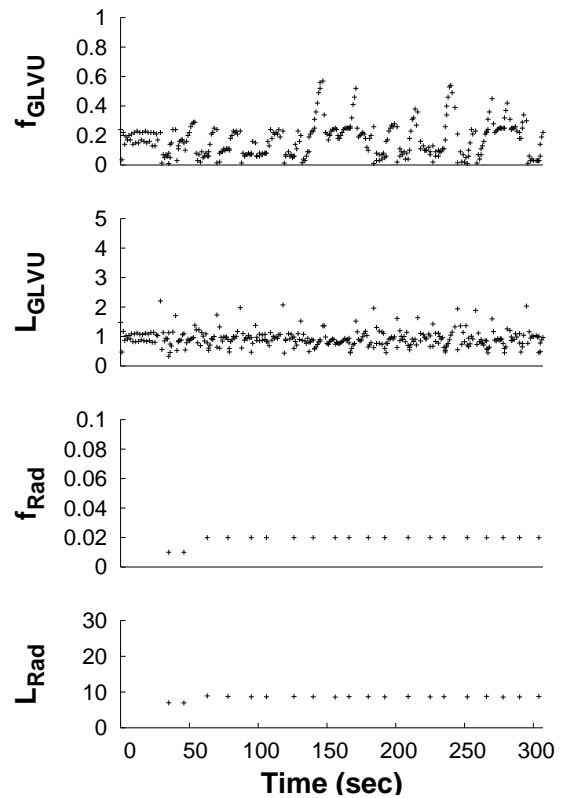

(a) Adaptive-both
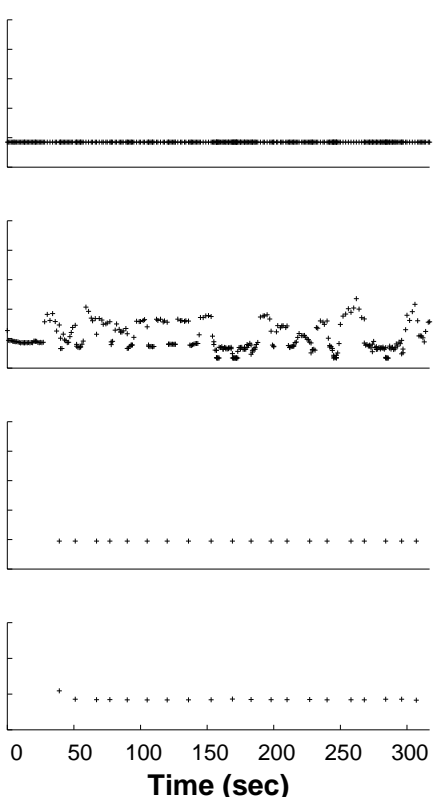

(b) Static-optimal
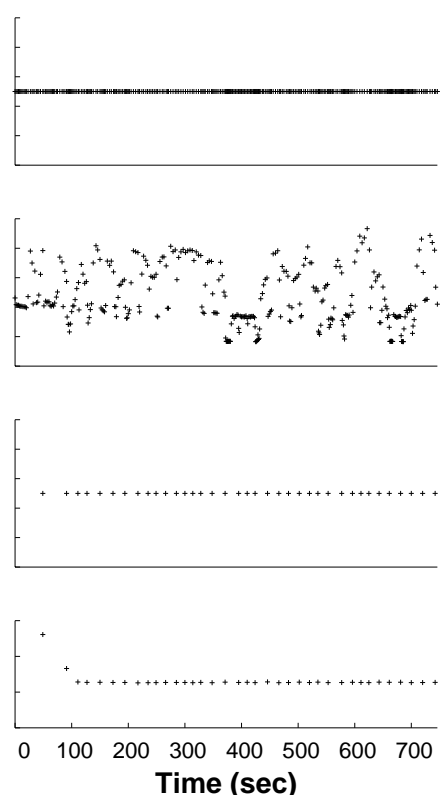

(c) Static-user

We show the performance of concurrent applications (GLVU and Radiator) over time, in three configurations. Each graph shows a time line of GLVU's fidelity $\left(f_{G L V U}\right)$, GLVU's latency in seconds $\left(L_{G L V U}\right)$, Radiator's fidelity $\left(f_{R a d}\right)$, and Radiator's latency in seconds $\left(L_{R a d}\right)$. Note the different time scale for the "static-user" graph. For lack of space, we omit the timelines for the "adaptive-GLVU" and "adaptive-Radiator" timelines: the adaptive and non-adaptive performance in these cases to that shown for the "adaptive-both" and "static-user" cases.

Figure 14: Adaptation in concurrent applications

as Linux processes at default priority.

GLVU replays a trace of a user navigating the virtual "Notre Dame" scene. Meanwhile, Radiator runs sporadic radiosity computations on a copy of the same scene in the background, to simulate occasional re-computation of lighting effects by the user. Between operations, Radiator sleeps for a random "think time" of $0-10 \mathrm{~s}$. The system's goal is to maintain the latency bounds of both applications despite resource variation. We use a $1 \mathrm{~s}$ latency bound for GLVU, as before. Radiator is much more resource-intensive, and runs in the background: for it, we use a $10 \mathrm{~s}$ bound.

We ran this experiment in 5 configurations:

- Adaptive-both: both applications adapt fidelity to achieve the target latency $(0.9 \mathrm{~s}$ for GLVU, $9 \mathrm{~s}$ for Radiator).

- Static-optimal: fidelity is static, but tuned for this benchmark. We set it to the mean fidelity achieved in the adaptive case ( 0.17 for GLVU, 0.019 for Radiator).

- Static-user: fidelity is static, at 0.5 for GLVU and 0.05 for Radiator: reasonable values that a user might select without workload-specific tuning.

- Adaptive-GLVU: GLVU adapts, Radiator uses the "staticuser" fidelity.

- Adaptive-Radiator: Radiator adapts, GLVU uses the "static-user" fidelity.
Figure 14 shows one trial each for the first three configurations. In the "adaptive-both" case, GLVU maintains its $1 \mathrm{~s}$ latency despite supply and demand variation. With "staticoptimal", mean latency is on target but variability is high; with "static-user", mean latency is off target and variability is even higher.

For Radiator, "adaptive-both" and "static-optimal" get latency on target, while "static-user" is off target. Variability is low in all cases: Radiator's CPU demand is invariant with time and camera position. CPU supply does not vary either: at $10 \mathrm{~s}$ time scales, the competing load imposed by GLVU is constant. "Static-optimal" has slightly lower variability than "adaptive", which executes a few operations at the wrong fidelity before converging on the correct value.

Figure 15 shows the mean normalized latency (latency divided by the latency bound) and coefficient of variation over 5 trials of all 5 configurations. We see that adaptation keeps latency on target without any workload-specific tuning, and reduces variation. Workload-specific tuning ("static-optimal") can get mean latency on target, but cannot prevent dynamic variation due to changes in resource supply or demand. Adaptation also insulates each application's performance from the other's: the "GLVU-only" and "Radiator-only" graphs show that the benefit gained from adaptation is independent of the other application's behavior. In other words, our approach 


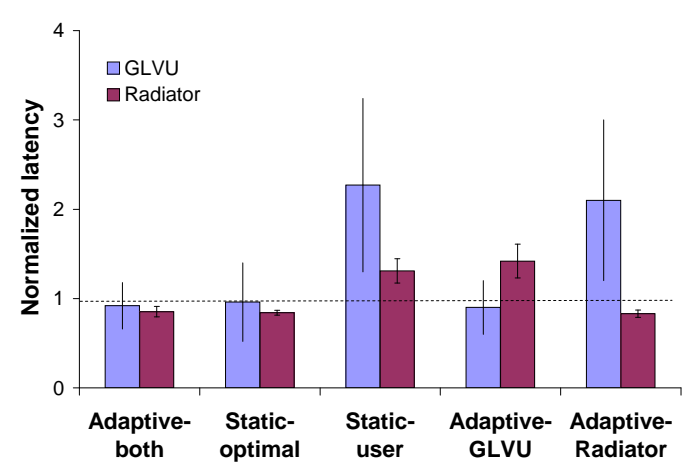

(a) Mean normalized latency

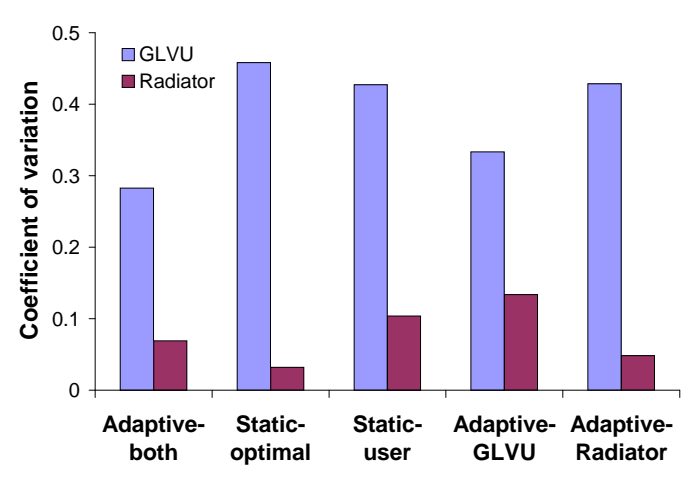

(b) Variability in latency

Error bars show standard deviations; the horizontal line marks the target latency.

Figure 15: Adaptive performance for concurrent applications

can be useful even without a coordinated effort to modify all running applications. This is a valuable property for realworld deployment.

\subsection{Costs and overheads}

\subsubsection{Porting costs}

The cost of porting legacy applications to a new API is an important measure of system deployability. Figure 16 shows the amount of source code modification required for four applications to use the multi-fidelity API. Three of these already had the potential for fidelity adaptation: for example, Radiator comes with support for multiresolution models. GLVU had to be augmented with multiresolution support, and we include the cost of this step.

Multi-fidelity support requires 500-1000 new or modified lines of code, including the ACF and hint module: a modest investment of programmer effort. Many of these lines are in glue code between application constructs and the generic

\begin{tabular}{|l|rr|rr|}
\hline \multirow{2}{*}{ Application } & \multicolumn{2}{|c|}{ Original size } & \multicolumn{2}{|c|}{ Modifications } \\
\cline { 2 - 5 } & KLOC & Files & KLOC & Files \\
\hline GLVU & 27.0 & 144 & $0.9^{\dagger}$ & $7^{\dagger}$ \\
Radiator & 51.1 & 222 & 0.6 & 5 \\
Web proxy & 3.9 & 9 & 0.9 & 6 \\
Speech recognizer & 126.4 & 209 & 1.1 & 10 \\
\hline
\end{tabular}

$\dagger$ Including multiresolution support (0.4 KLOC, 2 files)

Figure 16: Cost of porting legacy code

\begin{tabular}{|l|r|}
\hline Component & Overhead \\
\hline App-Odyssey communication & $0.36 \mathrm{~ms}$ \\
Logger (buffered at user level) & $0.15 \mathrm{~ms}$ \\
Logger (unbuffered) & $0.20 \mathrm{~ms}$ \\
CPU supply/demand monitor & $1.38 \mathrm{~ms}$ \\
Memory supply/demand monitor & $6.72 \mathrm{~ms}$ \\
Solver & $10.56 \mathrm{~ms}$ \\
\hline Total & $19.37 \mathrm{~ms}$ \\
\hline
\end{tabular}

Figure 17: Per-operation runtime overhead

multi-fidelity API. We are investigating the use of stub generators to automatically generate this glue code.

\subsubsection{Runtime overheads}

Figure 17 shows the overhead of each runtime component in additional latency per operation for a synthetic benchmark. The total overhead is around $20 \mathrm{~ms}$ : only $2 \%$ for a $1 \mathrm{~s}$ operation, but an unacceptable $20 \%$ for a $100 \mathrm{~ms}$ latency bound. We are looking at reducing the overheads by using a more efficient and scalable solver; with better interfaces for load and resource statistics (/proc contributes most of the latency to our resource predictors); and by replacing the middleware server with a library implementation.

\section{Related work}

This work is most closely related to previous work on fidelity adaptation [13, 27, 12, 7]. We have generalized these previous notions of fidelity, which only measured data degradation, to include arbitrary runtime parameters of an application. Our system and API also move the burden of adaptation out of the application: where other systems expect applications to specify their resource requirements, we predict resource supply, demand, and performance based on observations of history. Although resource demand prediction is still application-specific, it has been cleanly separated from the rest of the system, and our history-based methodology and measurement/logging infrastructure make it an easier task than before.

We also diverge from traditional models of adaptation by using a predictive rather than a feedback-driven approach. 
Rather than adjusting fidelity in small steps in response to a change in performance, Odyssey can make large yet accurate adaptations in a single step. This is made possible by Odyssey's ability to predict supply, demand and performance across the entire range of fidelities.

Related, but complementary to application adaptation is work on QoS-based reservations [23] and remote execution [11]: Section 2.1 discussed these in more detail.

Also related is previous work on resource prediction. Supply prediction - predicting load from past measurements is present in many systems. Usually it is present implicitly in a feedback loop: measurements of load or performance are used as control signals to adjust system parameters [32]. A few systems use explicit prediction of load: for example, Dinda's Running Time Advisor [8]. Explicit prediction of resource demand, however, is comparatively rare. Most systems assume that resource demand is constant, specified by the application, derived from a static probability distribution $[20,16]$, or obtained from compile-time analysis [31].

We know of two systems that explicitly predict resource demand as a function of runtime parameters: however, neither uses the predictions for application adaptation. Automated profiling for QoS [1] estimates the CPU utilization of a multimedia stream as a linear function of task rate and task size, for admission control purposes. PUNCH [18] uses machine learning to predict CPU demand as a function of application-specific runtime parameters, for load-balancing in a grid framework. To the best of our knowledge, Odyssey is the first system to use history-based prediction to model resource demand as a function of fidelity in adaptive applications.

\section{Conclusion}

We have shown in this paper that multi-fidelity computation supported by predictive resource management can improve performance in mobile interactive applications. Our performance evaluation shows that

- We reduce mean latency by $60 \%$ and variability by $30 \%$ for GLVU subjected to a time-varying load.

- History-based demand prediction is accurate and effective, with prediction errors as low as $0.3 \%$ for some resources and never higher than $24 \%$ in our case studies.

- The cost of using Odyssey is modest, involving 5001000 additional lines of code per application and $20 \mathrm{~ms}$ of runtime overhead per interactive operation.

Throughout the paper, we have indicated areas for incremental improvement; here we mention a few medium to longterm goals for future research. We would like to test Odyssey with a full-fledged AR application on wearable hardware, including location tracking and machine vision as well as rendering, and $100 \mathrm{~ms}$ latency bounds rather than $1 \mathrm{~s}$. We would like to further automate the construction of demand predictors: for example, by building platform-independent
CPU predictors that can be used across processor architectures. We would like to combine demand prediction with with QoS-based allocation such that the system can simultaneously optimize allocation across, and adaptation within, applications [28]. Finally, we would like to explore mixedinitiative [17] approaches that combine direct user modification of utility functions with automated inference by the system about user preferences.

\section{References}

[1] T. F. Abdelzaher. An automated profiling subsystem for QoSaware services. In Proc. Sixth IEEE Real-Time Technology and Applications Symposium (RTAS '00), pages 208-217, Washington, DC, June 2000.

[2] A. Arpaci-Dusseau and R. Arpaci-Dusseau. Information and control in gray-box systems. In Proc. 18th ACM Symposium on Operating Systems Principles (SOSP 2001), pages 43-56, Chateau Lake Louise, Banff, Canada, Oct. 2001.

[3] R. Azuma, Y. Baillot, R. Behringer, S. Feiner, S. Julier, and B. MacIntyre. Recent advances in augmented reality. IEEE Computer Graphics and Applications, 21(6):34-47, Nov./Dec. 2001.

[4] R. Balan, J. Flinn, M. Satyanarayanan, S. Sinnamohideen, and H.-I. Yang. The case for cyber foraging. In Proc. 10th ACM SIGOPS European Workshop, Saint-Emilion, France, Sept. 2002.

[5] M. Billinghurst, S. Weghorst, and T. A. Furness. Wearable computers for three dimensional CSCW. In Proc. International Symposium on Wearable Computers, pages 39-46, Cambridge, MA, Oct. 1997.

[6] M. F. Cohen and J. R. Wallace. Radiosity and Realistic Image Synthesis. Academic Press Professional, Boston, MA, 1993.

[7] E. de Lara, D. S. Wallach, and W. Zwaenepoel. Puppeteer: Component-based adaptation for mobile computing. In Proc. 3rd USENIX Symposium on Internet Technologies and Systems (USITS-01), pages 159-170, Berkeley, CA, Mar. 2001.

[8] P. A. Dinda. Online prediction of the running time of tasks. In Proc. 10th IEEE International Symposium on High Performance Distributed Computing (HPDC '01), pages 383-394, San Francisco, CA, Aug. 2001.

[9] A. H. Dutoit, O. Creighton, G. Klinker, R. Kobylinski, C. Vilsmeier, and B. Bruegge. Architectural issues in mobile augmented reality systems: a prototyping case study. In Proc. Eighth Asian Pacific Conference on Software Engineering (APSEC'2001), pages 341-344, Macau, China, Dec. 2001.

[10] S. Feiner, B. MacIntyre, T. Höllerer, and A. Webster. A Touring Machine: Prototyping 3D mobile augmented reality systems for exploring the urban environment. In Proc. International Symposium on Wearable Computers, pages 74-81, Cambridge, MA, Oct. 1997.

[11] J. Flinn, D. Narayanan, and M. Satyanarayanan. Self-tuned remote execution for pervasive computing. In Proc. 8th Workshop on Hot Topics in Operating Systems (HotOS-VIII), pages 61-66, Schloss Elmau, Germany, May 2001. 
[12] J. Flinn and M. Satyanarayanan. Energy-aware adaptation for mobile applications. In Proc. 17th ACM Symposium on Operating Systems Principles (SOSP '99), pages 48-63, Kiawah Island, SC, Dec. 1999.

[13] A. Fox, S. D. Gribble, E. A. Brewer, and E. Amir. Adapting to network and client variability via on-demand dynamic distillation. In Proc. 7th International Conference on Architectural Support for Programming Languages and Operating Systems (ASPLOS '96), pages 160-170, Cambridge, MA, Oct. 1996.

[14] M. Garland and P. S. Heckbert. Surface simplification using quadric error metrics. In Proc. SIGGRAPH '97, pages 209216, Los Angeles, CA, Aug. 1997.

[15] C. F. Gauss. Theoria Combinationis Observationum Erroribus Minimum Obnoxiae. Royal Society of Göttingen, 1821.

[16] M. Harchol-Balter and A. B. Downey. Exploiting process lifetime distributions for dynamic load balancing. In Proc. Joint International Conference on Measurement and Modeling of Computer Systems (ACM SIGMETRICS '94), pages 13-24, Nashville, TN, May 1994.

[17] E. Horvitz. Principles of mixed-initiative user interfaces. In Proc. ACM SIGCHI Conference on Human Factors in Computing Systems (CHI '99), pages 159-166, Pittsburgh, PA, May 1999.

[18] N. H. Kapadia, J. A. B. Fortes, and C. E. Brodley. Predictive application-performance modeling in a computational grid environment. In Proc. 8th IEEE International Symposium on High Performance Distributed Computing (HPDC '99), pages 47-54, Los Angeles, CA, Aug. 1999.

[19] C. Lee, J. Lehoczky, D. Siewiorek, R. Rajkumar, and J. Hansen. A scalable solution to the multi-resource QoS problem. In Proc. 20th IEEE Real-Time Systems Symposium (RTSS '99), pages 315-326, Phoenix, AZ, Dec. 1999.

[20] W. E. Leland and T. J. Ott. Load-balancing heuristics and process behavior. In Proc. Joint International Conference on Measurement and Modeling of Computer Systems (ACM SIGMETRICS '86), pages 54-69, Raleigh, NC, May 1986.

[21] R. B. Miller. Response time in man-computer conversational transactions. AFIPS Fall Joint Computer Conference Proceedings, 33:267-277, Dec. 1968.

[22] D. J. Musliner, E. H. Durfee, and K. G. Shin. Any-dimension algorithms. In Proc. 9th IEEE Workshop on Real-Time Operating Systems and Software (RTOSS '92), pages 78-81, May 1992.

[23] K. Nahrstedt, D. Xu, D. Wichadukul, and B. Li. QoS-aware middleware for ubiquitous and heterogeneous environments. IEEE Communications, 39(11):140-148, Nov. 2001.

[24] D. Narayanan. Operating System Support for Mobile Interactive Applications. PhD thesis, Carnegie Mellon University, Aug. 2002.

[25] D. Narayanan, J. Flinn, and M. Satyanarayanan. Using history to improve mobile application adaptation. In Proc. $3 r d$ IEEE Workshop on Mobile Computing Systems and Applicatons, pages 31-40, Monterey, CA, Dec. 2000.

[26] C. Narayanaswami, N. Kamijoh, M. Raghunath, T. Inoue, T. Cipolla, J. Sanford, E. Schlig, S. Venkiteswaran, D. Guniguntala, V. Kulkarni, and K. Yamazaki. IBM's Linux watch, the challenge of miniaturization. IEEE Computer, 35(1):3341, Jan. 2002.

[27] B. D. Noble, M. Satyanarayanan, D. Narayanan, J. E. Tilton, J. Flinn, and K. R. Walker. Agile application-aware adaptation for mobility. In Proc. 16th ACM Symposium on Operating Systems Principles (SOSP '97), pages 276-287, Saint Malo, France, Oct. 1997.

[28] D. Petrou and D. Narayanan. Position summary: Hinting for goodness' sake. In Proc. 8th Workshop on Hot Topics in Operating Systems (HotOS-VIII), page 177, Schloss Elmau, Germany, May 2001.

[29] T. W. Project. GLVU source code and online documentation. http://www.cs.unc.edu/ ${ }^{\sim}$ walk/software/glvu/, Feb. 2002.

[30] M. Satyanarayanan and D. Narayanan. Multi-fidelity algorithms for interactive mobile applications. Wireless Networks, 7:601-607, 2001.

[31] B. S. Siegell and P. Steenkiste. Automatic generation of parallel programs with dynamic load balancing. In Proc. 3rd IEEE International Symposium on High Performance Distributed Computing (HPDC '94), pages 166-175, San Francisco, CA, Aug. 1994.

[32] D. C. Steere, A. Goel, J. Gruenberg, D. McNamee, C. Pu, and J. Walpole. A feedback-driven proportion allocator for realrate scheduling. In Proc. 3rd Symposium on Operating Systems Design and Implementation (OSDI '99), pages 145-158, New Orleans, LA, Feb. 1999.

[33] G. K. Wallace. The JPEG still picture compression standard. Communications of the ACM, 34(4):30-44, Apr. 1991.

[34] A. Webster, S. Feiner, B. MacIntyre, W. Massie, and T. Krueger. Augmented reality in architectural construction, inspection and renovation. In Proc. ASCE Third Congress on Computing in Civil Engineering, pages 913-919, Anaheim, CA, June 1996.

[35] A. J. Willmott. Radiator source code and online documentation. http://www.cs.cmu.edu/ ${ }^{a j w / s o f t w a r e /, ~ O c t . ~}$ 1999.

[36] P. Young. Recursive Estimation and Time-Series Analysis. Springer, 1984. 